\title{
Incorporating conditional survival into prognostication for gunshot wounds to the head
}

\author{
${ }^{*}$ Patrick D. Kelly, MD, MSCl, ${ }^{1}$ Pious D. Patel, BA, ${ }^{2}$ Aaron M. Yengo-Kahn, MD, ${ }^{1}$ \\ Daniel I. Wolfson, MD, ${ }^{2}$ Fakhry Dawoud, BS, ${ }^{1,3}$ Ranbir Ahluwalia, BS, ${ }^{1,4}$ \\ Oscar D. Guillamondegui, MD, MPH, ${ }^{5}$ and Christopher M. Bonfield, MD1
}

\begin{abstract}
${ }^{1}$ Department of Neurological Surgery, Vanderbilt University Medical Center, Nashville; ${ }^{2}$ Vanderbilt University School of Medicine, Nashville; ${ }^{3}$ Quillen College of Medicine, East Tennessee State University, Mountain Home, Tennessee; ${ }^{4}$ College of Medicine, Florida State University, Tallahassee, Florida; and ${ }^{5}$ Division of Trauma, Emergency Surgery, and Critical Care, Vanderbilt University Medical Center, Nashville, Tennessee
\end{abstract}

OBJECTIVE Several scores estimate the prognosis for gunshot wounds to the head (GSWH) at the point of hospital admission. However, prognosis may change over the course of the hospital stay. This study measures the accuracy of the Baylor score among patients who have already survived the acute phase of hospitalization and generates conditional outcome curves for the duration of hospital stay for patients with GSWH.

METHODS Patients in whom GSWH with dural penetration occurred between January 2009 and June 2019 were identified from a trauma registry at a level I trauma center in the southeastern US. The Baylor score was calculated using component variables. Conditional overall survival and good functional outcome (Glasgow Outcome Scale score of 4 or 5) curves were generated. The accuracy of the Baylor score in predicting mortality and functional outcome among acutephase survivors (survival $>48$ hours) was assessed using receiver operating characteristic curves and the area under the curve (AUC).

RESULTS A total of 297 patients were included (mean age 38.0 [SD 15.7] years, 73.4\% White, 85.2\% male), and 129 patients survived the initial 48 hours of admission. These acute-phase survivors had a decreased mortality rate of $32.6 \%$ ( $n=42)$ compared to $68.4 \%(n=203)$ for all patients, and an increased rate of good functional outcome $(48.1 \% ; n=62)$ compared to the rate for all patients $(23.2 \% ; n=69)$. Among acute-phase survivors, the Baylor score accurately predicted mortality $(A \cup C=0.807$ ) and functional outcome $(A \cup C=0.837)$. However, the Baylor score generally overestimated true mortality rates and underestimated good functional outcome. Additionally, hospital day 18 represented an inflection point of decreasing probability of good functional outcome.

CONCLUSIONS During admission for GSWH, surviving beyond the acute phase of 48 hours doubles the rates of survival and good functional outcome. The Baylor score maintains reasonable accuracy in predicting these outcomes for acute-phase survivors, but generally overestimates mortality and underestimates good functional outcome. Future prognostic models should incorporate conditional survival to improve the accuracy of prognostication after the acute phase.

https://thejns.org/doi/abs/10.3171/2020.9.JNS202723

KEYWORDS GSW; gunshot wound; penetrating brain injury; prognostic scores; conditional survival; functional outcome; trauma; traumatic brain injury

$\mathrm{G}$ UNSHOT wounds to the head (GSWH), with approximately 20,000 annual cases, are the leading cause of traumatic brain injury (TBI) deaths in the US. ${ }^{1}$ GSWH carry a bleak prognosis, with studies showing a low survival rate beyond the emergency department (ED). ${ }^{2}$ The accurate estimation of prognosis remains a clinical priority, allowing the necessary allocation of resources and appropriate conversations with clinical support teams. Several studies have identified individual predictors for GSWH mortality and long-term functional outcome..$^{3-8}$

Composite prognostic scoring systems have been developed from predictors of GSWH outcomes. The Baylor

ABBREVIATIONS AUC = area under the curve; $E D=$ emergency department; EVD = external ventricular drain; GCS = Glasgow Coma Scale; GOS = Glasgow Outcome Scale; GSWH = gunshot wounds to the head; ICP = intracranial pressure; INR = international normalized ratio; ISS = Injury Severity Score; ROC = receiver operating characteristic; SPIN = Surviving Penetrating Injury to the Brain; TBI = traumatic brain injury.

SUBMITTED July 13, 2020. ACCEPTED September 8, 2020.

INCLUDE WHEN CITING Published online March 9, 2021; DOI: 10.3171/2020.9.JNS202723.

${ }^{*}$ P.D.K. and P.D.P. contributed equally to this work and share first authorship. 
score $^{9}$ is a system designed to predict mortality and functional outcome at 6 months as measured by the Glasgow Outcome Scale (GOS). ${ }^{10}$ This score provides an estimation of these outcomes through the use of 4 variables that are available at the time of admission: bullet trajectory, patient age, Glasgow Coma Scale (GCS) score, and pupillary response. This score benefits from an ease of use and accessibility not found among alternative scoring systems that have been published, such as the more complex Surviving Penetrating Injury to the Brain (SPIN) score. ${ }^{11-13}$ However, neither the Baylor score nor any of these alternative scoring systems have incorporated features of conditional survival during the inpatient stay, because they were designed for use at initial patient evaluation.

Conditional survival is defined as the probability of survival as a function of the amount of time a patient has already survived. This concept is often used to explain how prognostic factors evolve over time. ${ }^{14}$ Whereas several scores have been developed to estimate GSWH prognosis at the point of ED arrival, there are no known scores of conditional survival analyses documenting how GSWH prognosis evolves over the course of an inpatient stay. This study aimed to produce a conditional survival curve for mortality and functional outcome following GSWH. The secondary aim was to assess the accuracy of the commonly used Baylor score in predicting outcomes among patients who have already survived the acute resuscitation period of 48 hours.

\section{Methods}

\section{Study Design}

A retrospective cohort study was designed using data collected from the institutional trauma registry of Vanderbilt University Medical Center, a level I trauma center in the southeastern US, between January 1, 2009, and June 30,2019 . The trauma registry contains all patient admis- sions for which either a trauma activation or admission to trauma surgery occurred at this institution. The study was approved by the institutional review board, and data accession and storage were performed in accordance with the Health Insurance Portability and Accountability Act (HIPAA).

\section{Patient Selection}

Patients were included if they were admitted to our institution for GSWH with dural penetration as identified by CT scan of the head. No patients meeting these criteria were excluded. Patient records were manually reviewed. Patients who survived more than 48 hours were grouped as "acute survivors." The acute phase of resuscitation was defined as the first 48 hours of admission in order to maximize specificity in identifying patients who survived the acute phase of trauma. This definition is further supported by a clear inflection point at this time for rates of good outcomes (Fig. 1).

\section{Clinical Management During Study Period}

Acute trauma management was performed according to advanced trauma life support and current resuscitation guidelines. ${ }^{15,16}$ Management of TBI was largely in accordance with Brain Trauma Foundation guidelines, ${ }^{17}$ including the following: 1) intracranial pressure (ICP) was monitored when there was no clear mass lesion requiring evacuation and the GCS score was 8 or below; 2) hyperosmolar therapy of $3 \%$ saline was administered as boluses and continuous intravenous infusion, titrated to an ICP < $20 \mathrm{~mm} \mathrm{Hg}$ with a maximum serum sodium concentration of $155 \mathrm{mEq} / \mathrm{L}$; and 3) decompressive craniectomy was performed for unilateral lesions with mass effect and correlated neurological deficits or medically refractory increases in ICP.

Central venous catheters and arterial lines were placed

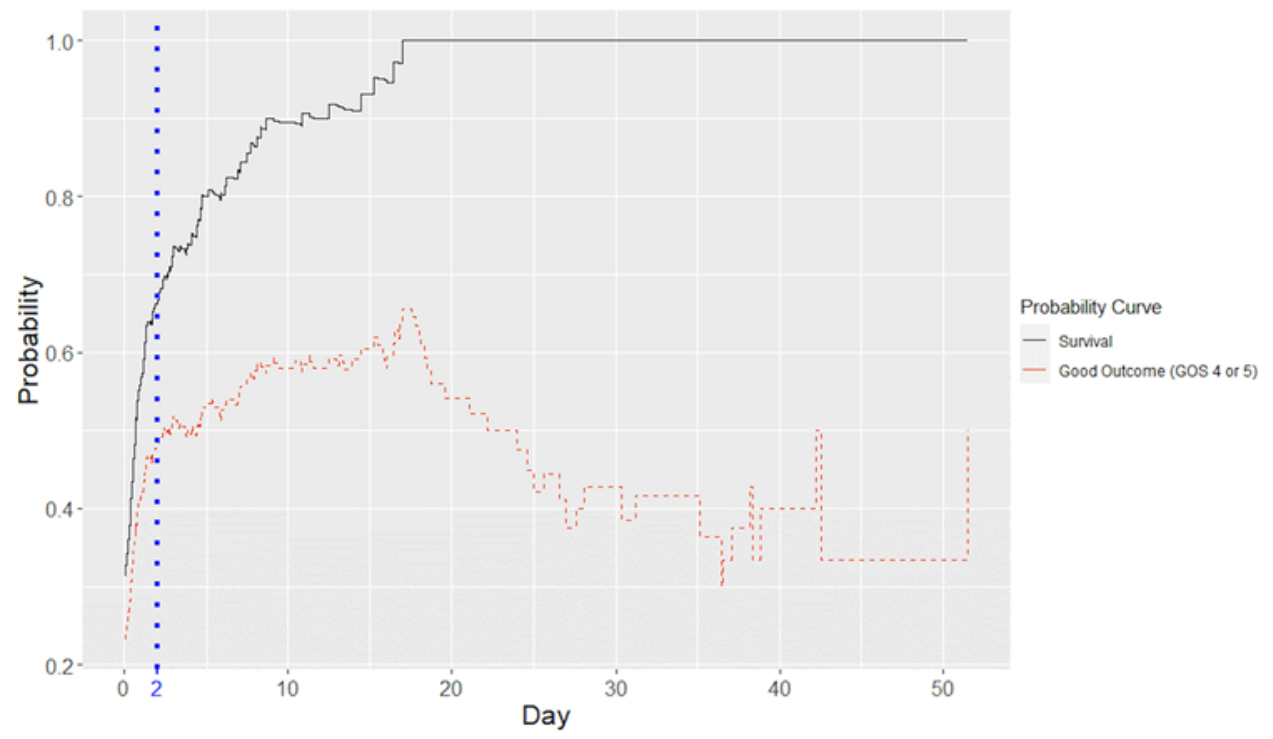

FIG. 1. Graph showing conditional survival and good functional outcome during hospital stay. Figure is available in color online only. 
routinely as indicated. During this time period, the institutional practice included placing an external ventricular drain (EVD) as the method of ICP monitoring when the ventricular size was appropriate, and to place an ICP monitor secondarily if the EVD failed or there was no accessible ventricle. Patients with coagulopathy received platelet transfusions for a goal of 100,000 platelets/ $\mu 1$, and fresh-frozen plasma or prothrombin complex concentrate was administered to maintain an international normalized ratio (INR) < 1.7. If the injury was thought to be nonsurvivable, as assessed by trauma and neurosurgery attending physicians' review of imaging and physical examination, consideration was given to shifting care toward end-of-life decision-making or cardiovascular support for organ donation. There was no minimum amount of time required before a patient's surrogate decision-maker could request palliative extubation or comfort care, and the clinical threshold for determining futility of care was determined by the attending physician of the primary team.

\section{Independent Variables}

For each patient, demographic information, medical history, admission vital signs, GCS score on admission, pupillary response on admission, bullet trajectory, laboratory studies, and imaging reports were collected from the medical record. The time from admission to death or discharge was calculated in hours based on the times of ED arrival and death or discharge. For bullet trajectory, a schema from previous studies was used to categorize this variable into 4 groups as follows: 1) unihemispheric or bifrontal; 2) bihemispheric; 3) posterior fossa; and 4) transventricular.,11 From the variables collected, the Baylor score was calculated using the following formula: (if age $>35$ years, then +1 ) + (if GCS score $=3$ or 4 , then $+1)+($ if nonreactive pupils bilaterally, then +1$)+($ if bullet trajectory posterior fossa or bihemispheric, then +2$){ }^{9}$ Higher scores are associated with worse outcomes, with total scores ranging from 0 to 5 . Estimated probabilities of mortality and good functional outcome corresponding to each score, as published by Gressot et al., are outlined in Table $1 .^{9}$

\section{Outcomes}

The primary outcome was survival to hospital discharge. The secondary outcome was functional outcome at the last follow-up. The functional outcome was measured in this study from the clinical note written for the patient's most recent follow-up. A GOS score from 1 to 5 was assigned from interpretation of the patient's history and physical examination. A GOS score of 1 corresponds to death, 2 corresponds to neurovegetative state, 3 corresponds to severe disability dependent on daily support, 4 corresponds to moderate disability but independence in daily life, and 5 corresponds to normal life with minor neurological deficits. ${ }^{10}$ Similarly to prior literature, a good functional outcome was defined as a GOS score of 4 or 5 at the last follow-up visit. ${ }^{9}$

\section{Statistical Analysis}

Categorical variables are presented as frequency and
TABLE 1. Probabilities of mortality and good functional outcome predicted by Baylor score

\begin{tabular}{ccc}
\hline & \multicolumn{2}{c}{ Outcomes at 6 Mos Postinjury } \\
\cline { 2 - 3 } Baylor Score & Mortality & Good Functional Outcome \\
\hline 0 & $25 \%$ & $50 \%$ \\
\hline 1 & & $30 \%$ \\
\hline 2 & $50 \%$ & $10 \%$ \\
\hline 3 & & $0 \%$ \\
\hline 4 & $75 \%$ & \\
\hline 5 & & 0 \\
\hline
\end{tabular}

Modified with permission from Gressot LV, Chamoun RB, Patel AJ, et al. Predictors of outcome in civilians with gunshot wounds to the head upon presentation. J Neurosurg. 2014;121(3):645-652.

proportion. Descriptive statistics for continuous variables are presented as mean (SD). For analysis, calculated Baylor scores were grouped based on the mortality and functional outcome correlates in the same manner as in Gressot et al. ${ }^{9}$ for mortality, the scores were grouped as $0-1$, 2 , and $3-5$, and for functional outcome, the scores were grouped as $0,1,2$, and 3-5.

Conditional survival curves for mortality and good functional outcome were plotted, with the $\mathrm{x}$-axis indicating the time survived and the y-axis charting the probability of the outcome conditional on having survived " $x$ " days. For the cohort of patients who survived the acutephase period of 48 hours after admission, receiver operating characteristic (ROC) curves were created and the area under the curve (AUC) was calculated for the ability of the Baylor score to discriminate mortality and good functional outcome.

Univariate analysis was performed to identify clinical variables associated with survival past the acute phase, as well as variables associated with mortality or good functional outcome within the population of acute-phase survivors. Nonparametric Wilcoxon rank-sum tests were used to compare differences in continuous variables between patients with or without the outcomes of interest. Fisher's exact tests were used to compare categorical variables. Statistical significance was set a priori at $\mathrm{p}<0.05$. Analyses were performed in Stata IC version 16.1 (StataCorp).

\section{Results}

\section{Baseline Characteristics}

A total of 297 patients were included (mean age 38.0 [SD 15.7] years, $73.4 \%$ White, $85.2 \%$ male). On admission, most patients had nonreactive pupils $(\mathrm{n}=177 ; 59.6 \%)$ and had GCS scores of 3 or $4(n=227 ; 76.4 \%)$. A total of 182 (61.3\%) patients had suicidal intent for GSWH. Overall, $52(17.5 \%)$ patients underwent craniotomy or craniectomy. A total of 203 (68.4\%) patients died during the hospital stay, whereas $69(23.2 \%)$ patients had a good functional outcome (Table 2).

\section{Conditional Survival and Functional Outcome}

Figure 1 shows conditional survival and good functional outcome. The time point of 48 hours represented a point 
TABLE 2. Baseline characteristics in 297 patients with GSWH

\begin{tabular}{|c|c|c|c|c|}
\hline Variable & $\begin{array}{l}\text { All Patients, } \\
n=297\end{array}$ & $\begin{array}{l}\text { Acute-Phase Survivors, Remained in Hospital } \\
\text { After } 48 \text { Hrs Admission, } n=129\end{array}$ & $\begin{array}{l}\text { Non-Acute-Phase } \\
\text { Survivors, } n=168\end{array}$ & $\mathrm{p}$ Value \\
\hline \multicolumn{5}{|l|}{ Demographics } \\
\hline Age in yrs, mean (SD) & $38.0(15.7)$ & $39.5(15.9)$ & $36.8(15.6)$ & 0.137 \\
\hline Race & & & & 0.2 \\
\hline Asian & $2(0.7 \%)$ & $2(1.6 \%)$ & $0(0.0 \%)$ & \\
\hline Black & $65(21.9 \%)$ & $33(25.6 \%)$ & $32(19.0 \%)$ & \\
\hline White & $218(73.4 \%)$ & $89(69.0 \%)$ & $129(76.8 \%)$ & \\
\hline Other & $12(4.0 \%)$ & $5(3.9 \%)$ & $7(4.2 \%)$ & \\
\hline Sex & & & & 0.647 \\
\hline Male & $253(85.2 \%)$ & $108(83.7 \%)$ & $145(86.3 \%)$ & \\
\hline Female & $44(14.8 \%)$ & $21(16.3 \%)$ & $23(13.7 \%)$ & \\
\hline \multicolumn{5}{|l|}{ Admission variables } \\
\hline Initial INR, mean (SD) & $1.7(1.5)$ & $1.2(0.2)$ & $2.2(1.9)$ & $<0.001$ \\
\hline MAP in mm Hg, mean (SD) & $98.98(25.40)$ & $102.6(22.7)$ & $95.7(27.4)$ & 0.04 \\
\hline ISS, mean (SD) & $25.3(7.9)$ & $24.3(8.6)$ & $26.2(7.3)$ & 0.04 \\
\hline Outside hospital transfer & $64(21.5 \%)$ & $28(21.7 \%)$ & $36(21.4 \%)$ & $>0.999$ \\
\hline Intent of injury & & & & 0.001 \\
\hline Suicide & $182(61.3 \%)$ & $71(55.0 \%)$ & $111(66.1 \%)$ & \\
\hline Homicide & $65(21.9 \%)$ & $41(31.8 \%)$ & $24(14.3 \%)$ & \\
\hline Accidental & $14(4.7 \%)$ & $8(6.2 \%)$ & $6(3.6 \%)$ & \\
\hline Unknown & $36(12.1 \%)$ & $9(7.0 \%)$ & $27(16.1 \%)$ & \\
\hline \multicolumn{5}{|l|}{ Midline shift } \\
\hline Present on CT scan & $114(38.4 \%)$ & $47(36.4 \%)$ & $67(39.9 \%)$ & 0.628 \\
\hline In mm, mean (SD) & $2.68(4.32)$ & $2.4(3.9)$ & $2.9(4.6)$ & 0.264 \\
\hline \multicolumn{5}{|l|}{ Bullet trajectory } \\
\hline Unihemispheric or bifrontal & $153(51.5 \%)$ & $105(81.4 \%)$ & $48(28.6 \%)$ & $<0.001$ \\
\hline Bihemispheric & $139(46.8 \%)$ & $21(16.3 \%)$ & $118(70.2 \%)$ & $<0.001$ \\
\hline Posterior fossa & $17(5.7 \%)$ & $6(4.7 \%)$ & $11(6.5 \%)$ & 0.656 \\
\hline Transventricular & $47(15.8 \%)$ & $12(9.3 \%)$ & $35(20.8 \%)$ & 0.011 \\
\hline GCS score & & & & $<0.001$ \\
\hline 3 or 4 & $227(76.4 \%)$ & $73(56.6 \%)$ & $154(91.7 \%)$ & \\
\hline $5-8$ & $13(4.4 \%)$ & $8(6.2 \%)$ & $5(3.0 \%)$ & \\
\hline$>8$ & $57(19.2 \%)$ & $48(37.2 \%)$ & $9(5.4 \%)$ & \\
\hline Pupil reactivity & & & & $<0.001$ \\
\hline None & $166(55.9 \%)$ & $21(16.3 \%)$ & $145(86.3 \%)$ & \\
\hline One & $20(6.7 \%)$ & $15(11.6 \%)$ & $5(3.0 \%)$ & \\
\hline Both & $76(25.6 \%)$ & $61(47.3 \%)$ & $15(8.9 \%)$ & \\
\hline Reactive + globe rupture & $22(7.4 \%)$ & $22(17.1 \%)$ & $0(0.0 \%)$ & \\
\hline Nonreactive + globe rupture & $11(3.7 \%)$ & $9(7.0 \%)$ & $2(1.2 \%)$ & \\
\hline Bilat globe rupture & $2(0.7 \%)$ & $1(0.8 \%)$ & $1(0.6 \%)$ & \\
\hline Baylor score & & & & $<0.001$ \\
\hline 0 & $28(9.4 \%)$ & $22(17.1 \%)$ & $6(3.6 \%)$ & \\
\hline 1 & $40(13.5 \%)$ & $36(27.9 \%)$ & $4(2.4 \%)$ & \\
\hline 2 & $67(22.6 \%)$ & $42(32.6 \%)$ & $25(14.9 \%)$ & \\
\hline 3 & $29(9.8 \%)$ & $16(12.4 \%)$ & $13(7.7 \%)$ & \\
\hline 4 & $72(24.2 \%)$ & $9(7.0 \%)$ & $63(37.5 \%)$ & \\
\hline 5 & $61(20.5 \%)$ & $4(3.1 \%)$ & $57(33.9 \%)$ & \\
\hline
\end{tabular}


TABLE 2. Baseline characteristics in 297 patients with GSWH

\begin{tabular}{|c|c|c|c|c|}
\hline Variable & $\begin{array}{l}\text { All Patients, } \\
n=297\end{array}$ & $\begin{array}{l}\text { Acute-Phase Survivors, Remained in Hospital } \\
\text { After } 48 \text { Hrs Admission, } n=129\end{array}$ & $\begin{array}{l}\text { Non-Acute-Phase } \\
\text { Survivors, } n=168\end{array}$ & p Value \\
\hline \multicolumn{5}{|l|}{ Treatments rendered } \\
\hline Coagulopathy correction performed & $87(29.3 \%)$ & $13(10.1 \%)$ & $74(44.0 \%)$ & $<0.001$ \\
\hline Craniotomy intervention performed & $52(17.5 \%)$ & $50(38.8 \%)$ & $2(1.2 \%)$ & $<0.001$ \\
\hline \multicolumn{5}{|l|}{ ICP treatments } \\
\hline EVD placed & $19(6.4 \%)$ & $18(14.0 \%)$ & $1(0.6 \%)$ & $<0.001$ \\
\hline ICP monitor placed & $14(4.7 \%)$ & $13(10.1 \%)$ & $1(0.6 \%)$ & $<0.001$ \\
\hline Max ICP in mm Hg, mean (SD) & $33.4(25.3)$ & $6.8(17.8)$ & $0.3(3.2)$ & $<0.001$ \\
\hline \multicolumn{5}{|l|}{ Outcome variables } \\
\hline In-hospital mortality & $203(68.4 \%)$ & $42(32.6 \%)$ & $161(95.8 \%)$ & $<0.001$ \\
\hline GOS score & & & & $<0.001$ \\
\hline 1 & $203(68.4 \%)$ & $42(32.6 \%)$ & $161(95.8 \%)$ & \\
\hline 2 & $2(0.7 \%)$ & $2(1.6 \%)$ & $0(0.0 \%)$ & \\
\hline 3 & $23(7.7 \%)$ & $23(17.8 \%)$ & $0(0.0 \%)$ & \\
\hline 4 & $24(8.1 \%)$ & $22(17.1 \%)$ & $2(1.2 \%)$ & \\
\hline 5 & $45(15.2 \%)$ & $40(31.0 \%)$ & $5(3.0 \%)$ & \\
\hline Length of stay in days, mean (SD) & $6.1(10.0)$ & $12.6(12.5)$ & $1.1(0.3)$ & $<0.001$ \\
\hline ICU length of stay in days, mean (SD) & $3.9(5.1)$ & $7.1(6.3)$ & $1.4(0.6)$ & $<0.001$ \\
\hline
\end{tabular}

$\mathrm{MAP}=$ mean arterial pressure; $\max =$ maximum .

Unless otherwise indicated, values are expressed as the number of patients (\%).

of significant change in conditional survival and functional outcome, with a total of 129 (43\%) patients surviving to this time point (acute-phase survivors). In comparison to the $168(57 \%)$ patients who died or were discharged prior to 48 hours, the acute-phase survivors had significantly decreased features of injury severity on admission and had higher rates of neurosurgical interventions during their stay (Table 2). Among the patients who did not remain in the hospital past 48 hours, there were $126(75 \%)$ who were declared brain dead, 33 (20\%) who suffered cardiac death despite maximum resuscitation efforts, and $4(2 \%)$ who had withdrawal of care without brain death. Whereas the overall mortality rate was $68.4 \%(n=203)$, the acutephase survivors experienced a reduced mortality rate of $32.6 \%(n=42)$. Whereas the overall rate of good functional outcome was $23.2 \%(n=69)$, the acute-phase survivors experienced an improved rate of good functional outcome of $48.1 \%(n=62)$. The time period of $18-25$ days, on the other hand, represented an inflection toward worse outcomes. The rates of good functional outcome were $66.7 \%$ $(\mathrm{n}=20)$ and $50.0 \%(\mathrm{n}=10)$ for patients who remained in the hospital at day 18 and at day 25 , respectively.

\section{Baylor Score Performance After Acute Phase}

Among acute-phase survivors, the Baylor score predicted mortality with an AUC of 0.7749 (Fig. 2A). The score groups $0-1,2$, and 3-5 overestimated true mortality rates. The Baylor score predicted good functional outcome with an AUC of 0.8146 (Fig. 2B). The score groups $0,1,2$, and 3-5 underestimated true rates of good functional outcome.

\section{Univariate Analyses}

Tables 3 and 4 show univariate associations of clinical variables with mortality and good functional outcome, respectively, among acute-phase survivors. Notably, mortality was associated with bihemispheric $(\mathrm{p}<0.001)$ or transventricular $(\mathrm{p}=0.002)$ bullet trajectories; increased age $(\mathrm{p}=0.027)$; nonreactive pupils on admission $(\mathrm{p}<$ $0.001)$; lower GCS score on admission ( $\mathrm{p}=0.019)$; degree of midline shift $(\mathrm{p}=0.005)$ or presence of herniation on CT scan $(p=0.004)$; presence of epidural $(p=0.010)$, subarachnoid $(p=0.008)$, or intraparenchymal $(p=0.007)$ hematoma; and absence of craniotomy intervention $(\mathrm{p}=$ 0.020). Poor functional outcome (GOS score of 1-3) for acute-phase survivors was associated with bihemispheric $(p<0.001)$ or transventricular $(p=0.005)$ bullet trajectories, increased age $(\mathrm{p}<0.001)$, nonreactive pupils on admission $(p<0.001)$, motor GCS score $(p=0.002)$, female $\operatorname{sex}(p=0.017)$, intraparenchymal hematoma $(p=0.037)$, and absence of craniotomy intervention $(\mathrm{p}=0.018)$.

\section{Discussion}

This study describes features of conditional survival for patients with GSWH during the inpatient stay and presents a key inflection point of 48 hours postadmission. Survival after the acute resuscitation phase, defined here as 48 hours, doubles the rates of survival and good functional outcome. These findings suggest that 48 hours is a reasonable time point to reevaluate prognosis following GSWH, for both management decisions and family counseling. 

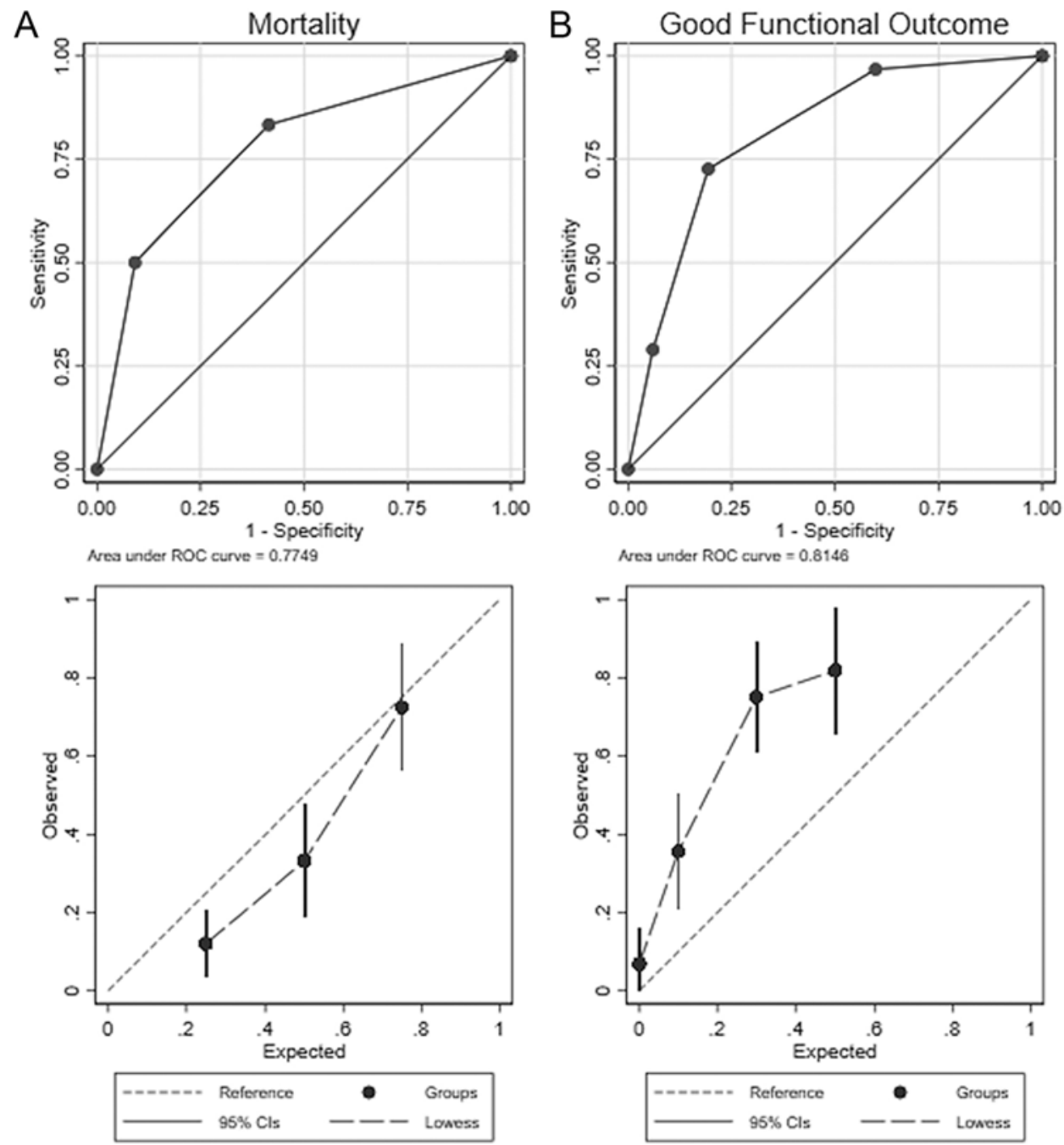

FIG. 2. A: The Baylor score's prediction of mortality among acute-phase survivors, illustrated by an ROC curve with AUC and calibration curve with 95\% Cls. B: The Baylor score's prediction of good functional outcome, defined as a GOS score of 4 or 5 at the last follow-up visit, among acute-phase survivors, illustrated by an ROC curve with AUC and calibration curve with $95 \%$ Cls. Lowess $=$ locally weighted scatterplot smoothing.

The accuracy of a commonly used prognostic score at ED admission, the Baylor score, was then assessed after this key inflection point of survival past 48 hours. For these acute-phase survivors, the Baylor score was found to be reasonably accurate, but it overestimated the odds of death while underestimating the odds of good functional outcome. In addition, hospital day 18 represents an inflection point of decreasing probability for good functional outcome.

Univariate analysis of acute-phase survivors was performed to assess for other important prognostic factors for the initial survivor population, which are not included in the Baylor score. This analysis revealed that good functional outcome, but not survival, is influenced by male sex. Survival, but not good functional outcome, is associated with a GCS score $>3$ or 4 on admission, as well as the absence of midline shift or herniation on head CT. Conversely, the Gressot study found that a low GCS score was significantly associated with good functional outcome, rather than survival. In addition, that study found neither survival nor functional outcome to be significantly influenced by sex, and its final Baylor score incorporated GCS score, but not sex, as a component variable. Similar comparisons between the univariate analyses in this study and those from the Gressot study may aid in the development of future GSWH prognostic models that incorporate conditional survival over the course of a patient's hospital stay.

The concept of conditional survival has been successfully applied toward long-term modeling of outcomes for chronic disease management. ${ }^{14}$ The integration of features representing survival up to a certain time point has led to more accurate prognostic estimates in several cancer disciplines, ${ }^{18-21}$ as well as for pathologies involving chronic organ failure. ${ }^{22,23}$ In acute care, conditional survival models have been used in intensive care settings to adjust mortality estimates for patients with longer stays ${ }^{24}$ For trauma care, time-based confounding has been identified as a major source of bias in outcome estimation. ${ }^{25}$ However, despite the importance of time-dependent factors in neu- 
TABLE 3. Univariate associations for survival to discharge among acute-phase survivors

\begin{tabular}{|c|c|c|c|}
\hline Variable & Survival to Discharge, $n=87$ & Nonsurvival, $n=42$ & $\mathrm{p}$ Value \\
\hline Unihemispheric or bifrontal trajectory & $81(93 \%)$ & $24(57 \%)$ & $<0.001$ \\
\hline Bihemispheric trajectory & $5(6 \%)$ & $16(38 \%)$ & $<0.001$ \\
\hline Posterior fossa trajectory & $3(3 \%)$ & $3(7 \%)$ & 0.390 \\
\hline Transventricular trajectory & $3(3 \%)$ & $9(21 \%)$ & 0.002 \\
\hline Age in yrs, median (IQR) & $31.00(23.00,53.00)$ & $45.00(29.00,58.00)$ & 0.027 \\
\hline Pupil reactivity & & & $<0.001$ \\
\hline None & $7(8 \%)$ & $14(33 \%)$ & \\
\hline One & $7(8 \%)$ & $8(19 \%)$ & \\
\hline Both & $47(54 \%)$ & $14(33 \%)$ & \\
\hline Reactive + globe rupture & $19(22 \%)$ & $3(7 \%)$ & \\
\hline Nonreactive + globe rupture & $7(8 \%)$ & $2(5 \%)$ & \\
\hline Bilat globe rupture & $0(0 \%)$ & $1(2 \%)$ & \\
\hline Max ICP in mm Hg, median (IQR) & $22.00(16.00,34.00), n=15$ & $33.00(21.00,95.00), n=11$ & 0.082 \\
\hline GCS score & & & 0.019 \\
\hline 3 & $41(47 \%)$ & $28(67 \%)$ & \\
\hline 4 & $1(1 \%)$ & $3(7 \%)$ & \\
\hline 5 & $1(1 \%)$ & $1(2 \%)$ & \\
\hline 6 & $3(3 \%)$ & $1(2 \%)$ & \\
\hline 7 & $1(1 \%)$ & $0(0 \%)$ & \\
\hline 8 & $1(1 \%)$ & $0(0 \%)$ & \\
\hline 9 & $2(2 \%)$ & $2(5 \%)$ & \\
\hline 10 & $1(1 \%)$ & $0(0 \%)$ & \\
\hline 11 & $5(6 \%)$ & $0(0 \%)$ & \\
\hline 12 & $2(2 \%)$ & $3(7 \%)$ & \\
\hline 13 & $1(1 \%)$ & $1(2 \%)$ & \\
\hline 14 & $7(8 \%)$ & $1(2 \%)$ & \\
\hline 15 & $21(24 \%)$ & $2(5 \%)$ & \\
\hline GCS score, motor component & & & 0.003 \\
\hline 1 & $41(47 \%)$ & $30(71 \%)$ & \\
\hline 2 & $2(2 \%)$ & $1(2 \%)$ & \\
\hline 3 & $0(0 \%)$ & $1(2 \%)$ & \\
\hline 4 & $6(7 \%)$ & $1(2 \%)$ & \\
\hline 5 & $6(7 \%)$ & $5(12 \%)$ & \\
\hline 6 & $32(37 \%)$ & $4(10 \%)$ & \\
\hline Male sex & $75(86 \%)$ & $33(79 \%)$ & 0.310 \\
\hline Initial INR, median (IQR) & $1.10(1.10,1.20), n=86$ & $1.20(1.10,1.30)$ & 0.140 \\
\hline Systolic blood pressure in ED in mm Hg, median (IQR) & $125.00(110.00,145.00), n=86$ & $121.00(105.00,145.00), n=40$ & 0.390 \\
\hline Degree of midline shift in mm, median (IQR) & $0.00(0.00,2.00)$ & $3.00(0.00,6.00)$ & 0.005 \\
\hline Herniation on CT scan & $6(7 \%)$ & $11(26 \%)$ & 0.004 \\
\hline Epidural hematoma on CT scan & $0(0 \%)$ & $4(10 \%)$ & 0.010 \\
\hline Subdural hematoma on CT scan & $49(56 \%)$ & $27(64 \%)$ & 0.450 \\
\hline Subarachnoid hematoma on CT scan & $60(69 \%)$ & $38(90 \%)$ & 0.008 \\
\hline Intraparenchymal hematoma on CT scan & $66(76 \%)$ & $40(95 \%)$ & 0.007 \\
\hline Craniotomy intervention performed & $40(46 \%)$ & $10(24 \%)$ & 0.020 \\
\hline
\end{tabular}

Unless otherwise indicated, values are expressed as the number of patients (\%).

rotrauma outcomes, conditional survival methods have not yet been applied in the GSWH population.

For GSWH, accurate estimation of prognosis remains a clinical priority in order to effectively inform care deci- sions in collaboration with surrogate decision-makers. Our analysis suggests that scores dependent on clinical factors measured at ED admission, including the Baylor score, may require adjustment based on conditional survival during 
TABLE 4. Univariate associations for good functional outcome among acute-phase survivors

\begin{tabular}{|c|c|c|c|}
\hline Variable & Bad Functional Outcome, $n=67$ & Good Functional Outcome, $n=62$ & p Value \\
\hline Unihemispheric or bifrontal trajectory & $46(69 \%)$ & $59(95 \%)$ & $<0.001$ \\
\hline Bihemispheric trajectory & $18(27 \%)$ & $3(5 \%)$ & $<0.001$ \\
\hline Posterior fossa trajectory & $5(7 \%)$ & $1(2 \%)$ & 0.210 \\
\hline Transventricular trajectory & $11(16 \%)$ & $1(2 \%)$ & 0.005 \\
\hline Age in yrs, median (IQR) & $46.00(28.00,58.00)$ & $28.50(23.00,46.00)$ & $<0.001$ \\
\hline Pupil reactivity & & & $<0.001$ \\
\hline None & $19(28 \%)$ & $2(3 \%)$ & \\
\hline One & $8(12 \%)$ & $7(11 \%)$ & \\
\hline Both & $26(39 \%)$ & $35(56 \%)$ & \\
\hline Reactive + globe rupture & $6(9 \%)$ & $16(26 \%)$ & \\
\hline Nonreactive + globe rupture & $7(10 \%)$ & $2(3 \%)$ & \\
\hline Bilateral globe rupture & $1(1 \%)$ & $0(0 \%)$ & \\
\hline Max ICP in mm Hg, median (IQR) & $26.00(18.00,37.00), n=17$ & $22.00(13.00,27.00), n=9$ & 0.240 \\
\hline GCS score & & & 0.100 \\
\hline 3 & $43(64 \%)$ & $26(42 \%)$ & \\
\hline 4 & $3(4 \%)$ & $1(2 \%)$ & \\
\hline 5 & $1(1 \%)$ & $1(2 \%)$ & \\
\hline 6 & $2(3 \%)$ & $2(3 \%)$ & \\
\hline 7 & $1(1 \%)$ & $0(0 \%)$ & \\
\hline 8 & $0(0 \%)$ & $1(2 \%)$ & \\
\hline 9 & $2(3 \%)$ & $2(3 \%)$ & \\
\hline 10 & $1(1 \%)$ & $0(0 \%)$ & \\
\hline 11 & $1(1 \%)$ & $4(6 \%)$ & \\
\hline 12 & $3(4 \%)$ & $2(3 \%)$ & \\
\hline 13 & $1(1 \%)$ & $1(2 \%)$ & \\
\hline 14 & $3(4 \%)$ & $5(8 \%)$ & \\
\hline 15 & $6(9 \%)$ & $17(27 \%)$ & \\
\hline GCS score, motor component & & & 0.002 \\
\hline 1 & $45(67 \%)$ & $26(42 \%)$ & \\
\hline 2 & $1(1 \%)$ & $2(3 \%)$ & \\
\hline 3 & $1(1 \%)$ & $0(0 \%)$ & \\
\hline 4 & $2(3 \%)$ & $5(8 \%)$ & \\
\hline 5 & $8(12 \%)$ & $3(5 \%)$ & \\
\hline 6 & $10(15 \%)$ & $26(42 \%)$ & \\
\hline Male sex & $51(76 \%)$ & $57(92 \%)$ & 0.017 \\
\hline Initial INR, median (IQR) & $1.20(1.10,1.30)$ & $1.10(1.00,1.20), n=61$ & 0.100 \\
\hline Systolic blood pressure in ED in $\mathrm{mm} \mathrm{Hg}$, median (IQR) & $122.00(110.00,145.00), n=64$ & $127.00(110.00,145.00)$ & 0.720 \\
\hline Degree of midline shift in mm, median (IQR) & $0.00(0.00,6.00)$ & $0.00(0.00,2.00)$ & 0.069 \\
\hline Herniation on CT scan & $12(18 \%)$ & $5(8 \%)$ & 0.120 \\
\hline Epidural hematoma on CT scan & $4(6 \%)$ & $0(0 \%)$ & 0.120 \\
\hline Subdural hematoma on CT scan & $39(58 \%)$ & $37(60 \%)$ & $>0.999$ \\
\hline Subarachnoid hematoma on CT scan & $55(82 \%)$ & $43(69 \%)$ & 0.100 \\
\hline Intraparenchymal hematoma on CT scan & $60(90 \%)$ & $46(74 \%)$ & 0.037 \\
\hline Craniotomy intervention performed & $19(28 \%)$ & $31(50 \%)$ & 0.018 \\
\hline
\end{tabular}

Unless otherwise indicated, values are expressed as the number of patients (\%).

the inpatient stay. For patients who survive more than 48 hours after admission, surrogate decision-makers should be informed of a potential underestimation of the likelihood of good outcome. They may also be informed of an overall 2-fold improvement in estimated outcome at this time point compared to the time of admission. This may be particularly helpful to correct potential overestimation of mortality among patients who have a high Baylor score 
on admission. This correction can be used to guide conversations and counseling with the patient's family, which may lead to altered goals of care and treatment decisions. After hospital day 17, surrogate decision-makers may be informed that the likelihood of a good functional outcome may begin to decrease with time. Chart review of these patients' treatment courses confirmed trends of injury complications, including brain abscesses, seizure disorders, pseudoaneurysm, and blindness, which can impact eventual functional outcome (data not shown). However, worsening outcomes for patients who remained admitted after day 17 may have been a result of selective censoring and may include patients who were more dependent, in which case the extended hospital stay would be a function of long-term care facility acceptance or other placement issues. Future studies may explore how systemic issues such as socioeconomic status, disposition barriers, and surrogate decision-maker conflicts can interact with outcomes after the acute phase of admission, such as length of stay.

Although the Baylor score performed reasonably well among acute-phase survivors, other scores with more complex components may perform better. The SPIN score is an alternative GSWH prognostic score, validated to predict survival only, which incorporates 5 additional features (sex, Injury Severity Score [ISS], INR, transfer status, and suicidal intent). ${ }^{11,26}$ These additional components may improve the granularity of survival prediction among acute-phase survivors, but rely on features that may not be immediately available at the time of presentation (e.g., ISS, INR, and suicidal intent). Regardless, they still do not incorporate conditional survival.

Both the Baylor score and SPIN score are designed to estimate outcomes at the point of ED admission, and future work may attempt to expand these scoring systems to estimate prognosis at later points during the inpatient stay. Decision-tree modeling may be a useful approach that allows incorporation of "survival by time $\mathrm{x}$ " as a logical branch point before considering time-dependent clinical variables. Prognostic decision-tree models have been developed for GSWH, but are currently limited by low sample size and omission of conditional survival features. ${ }^{12}$ Although this study would be underpowered to develop a validated GSWH prognostic model incorporating conditional survival, Tables 3 and 4 present univariate associations of several clinically relevant variables with outcomes among the acute-phase survivor population. Future GSWH prognostic models that aim to incorporate conditional survival may use these findings to guide model design.

\section{Limitations}

This analysis was performed at a single, level I trauma center in the southeastern US, with a predominantly White population. This limits the study's generalizability, inviting a future study incorporating data from multiple institutions. As a retrospective cohort study, there was a limitation in the ability to control for confounding medical conditions, treatment decisions, and clinical protocols. Furthermore, the input of family and surrogate decisionmakers regarding transitions to comfort care could not be ascertained from the medical record and thus was not in- corporated into this data set. Although we have described the trauma and neurosurgical management guidelines in place at our institution during the study period, it is important to acknowledge the nuanced nature of trauma care dependent on individual providers' decisions and decisionmaker input. In addition, the measurement of how variable and uncontrollable aspects of patient care during the GSWH inpatient stay affect prognostic estimates was a core component of our study question. The definition of acute phase as the first 48 hours after admission was an arbitrary choice designed to maximize specificity when identifying acute-phase survivors. Although an alternative definition (e.g., 24 hours) may have yielded different results, this definition is often used in the TBI literature, ${ }^{27-29}$ with a 1980 epidemiology study showing most head injury deaths occurring in the first 48 hours. ${ }^{30}$ Furthermore, the 48-hour point may serve as a more realistic time for clinical teams to have an updated prognostication discussion. The nonstandardized time points for outpatient follow-up may be an additional source of variability in our measurements, because the Baylor score was designed to predict the GOS score at 6 months after discharge. Future studies may be designed with longer follow-up periods to minimize the underestimation of good functional outcome.

\section{Conclusions}

For patients sustaining GSWH, survival beyond the acute phase of 48 hours after admission doubles the rate of survival and good functional outcome. Prognostication for acute-phase survivors by using existing predictive scores maintains a reasonable estimation of mortality and functional outcome but, importantly, underestimates good outcomes. These findings can refine estimates of mortality and functional outcome during prognostication discussion at the 48-hour time point. Univariate associations are provided in this study. The relationships presented between clinical variables with mortality and functional outcome among acute-phase survivors may be used to develop future conditional survival prognostic models for GSWH. Clinicians should recognize the need to adjust prognosis scores with features of conditional survival, and 48 hours appears to be an appropriate time point for reevaluating prognosis.

\section{Acknowledgments}

Sources of support include the following: REDCap, used for study data management, is supported by NCATS/NIH grant no. UL1 TR000445. Dr. Kelly is supported by a training grant from the National Cancer Institute of the NIH under award no. T32CA106183.

\section{References}

1. Alao T, Waseem M. Penetrating head trauma. In: StatPearls. StatPearls Publishing; 2020. Accessed November 4, 2020. https://www.ncbi.nlm.nih.gov/books/NBK459254/

2. Alvis-Miranda HR, Adie Villafañe R, Rojas A, et al. Management of craniocerebral gunshot injuries: a review. Korean J Neurotrauma. 2015;11(2):35-43.

3. Bandt SK, Greenberg JK, Yarbrough CK, et al. Management of pediatric intracranial gunshot wounds: predictors of favorable clinical outcome and a new proposed treatment paradigm. J Neurosurg Pediatr. 2012;10(6):511-517. 
4. Souter MJ, Blissitt PA, Blosser S, et al. Recommendations for the critical care management of devastating brain injury: prognostication, psychosocial, and ethical management: a position statement for healthcare professionals from the Neurocritical Care Society. Neurocrit Care. 2015;23(1):4-13.

5. Kim KA, Wang MY, McNatt SA, et al. Vector analysis correlating bullet trajectory to outcome after civilian throughand-through gunshot wound to the head: using imaging cues to predict fatal outcome. Neurosurgery. 2005;57(4):737-747.

6. Martins RS, Siqueira MG, Santos MTS, et al. Prognostic factors and treatment of penetrating gunshot wounds to the head. Surg Neurol. 2003;60(2):98-104.

7. Aarabi B, Tofighi B, Kufera JA, et al. Predictors of outcome in civilian gunshot wounds to the head. J Neurosurg. 2014; 120(5):1138-1146.

8. Aldrich EF, Eisenberg HM, Saydjari C, et al. Predictors of mortality in severely head-injured patients with civilian gunshot wounds: a report from the NIH Traumatic Coma Data Bank. Surg Neurol. 1992;38(6):418-423.

9. Gressot LV, Chamoun RB, Patel AJ, et al. Predictors of outcome in civilians with gunshot wounds to the head upon presentation. J Neurosurg. 2014;121(3):645-652.

10. Teasdale GM, Pettigrew LE, Wilson JT, et al. Analyzing outcome of treatment of severe head injury: a review and update on advancing the use of the Glasgow Outcome Scale. J Neurotrauma. 1998;15(8):587-597.

11. Muehlschlegel S, Ayturk D, Ahlawat A, et al. Predicting survival after acute civilian penetrating brain injuries: the SPIN score. Neurology. 2016;87(21):2244-2253.

12. Kim LH, Quon JL, Cage TA, et al. Mortality prediction and long-term outcomes for civilian cerebral gunshot wounds: a decision-tree algorithm based on a single trauma center. $J$ Clin Neurosci. 2020;75:71-79.

13. Tunthanathip T, Udomwitthayaphiban S. Development and validation of a nomogram for predicting the mortality after penetrating traumatic brain injury. Bull Emerg Trauma. 2019; 7(4):347-354.

14. Hieke S, Kleber M, König C, et al. Conditional survival: a useful concept to provide information on how prognosis evolves over time. Clin Cancer Res. 2015;21(7):1530-1536.

15. ATLS Subcommittee, American College of Surgeons' Committee on Trauma, International ATLS Working Group. Advanced trauma life support (ATLS): the ninth edition. $J$ Trauma Acute Care Surg. 2013;74(5):1363-1366.

16. Henry S. ATLS 10th edition offers new insights into managing trauma patients. Bulletin of the American College of Surgeons. June 1, 2018. Accessed November 4, 2020. https: //bulletin.facs.org/2018/06/atls-10th-edition-offers-newinsights-into-managing-trauma-patients/

17. Carney N, Totten AM, O'Reilly C, et al. Guidelines for the Management of Severe Traumatic Brain Injury, Fourth Edition. Neurosurgery. 2017;80(1):6-15.

18. Skuladottir H, Olsen JH. Conditional survival of patients with the four major histologic subgroups of lung cancer in Denmark. J Clin Oncol. 2003;21(16):3035-3040.

19. Zamboni BA, Yothers G, Choi M, et al. Conditional survival and the choice of conditioning set for patients with colon cancer: an analysis of NSABP trials C-03 through C-07. J Clin Oncol. 2010;28(15):2544-2548.

20. Zabor EC, Gonen M, Chapman PB, Panageas KS. Dynamic prognostication using conditional survival estimates. Cancer. 2013;119(20):3589-3592.

21. Seow H, Tanuseputro P, Barbera L, et al. Development and validation of a prognostic survival model with patient-reported outcomes for patients with cancer. JAMA Netw Open. 2020;3(4):e201768.
22. Efird JT, O’Neal WT, Camargo GA, et al. Conditional survival of heart failure patients after coronary artery bypass grafting. J Cardiovasc Med (Hagerstown). 2014;15(6):498-503.

23. Zheng M-H, Wu S-J, Shi K-Q, et al. Conditional survival estimate of acute-on-chronic hepatitis B liver failure: a dynamic prediction based on a multicenter cohort. Oncotarget. 2015;6(27):23261-23271.

24. Marshall DC, Hatch RA, Gerry S, et al. Conditional survival with increasing duration of ICU admission: an observational study of three intensive care databases. Crit Care Med. 2020; 48(1):91-97.

25. del Junco DJ, Fox EE, Camp EA, et al. Seven deadly sins in trauma outcomes research: an epidemiologic post mortem for major causes of bias. J Trauma Acute Care Surg. 2013;75(1) (suppl 1):S97-S103.

26. Mikati AG, Flahive J, Khan MW, et al. Multicenter validation of the Survival After Acute Civilian Penetrating Brain Injuries (SPIN) Score. Neurosurgery. 2019;85(5):E872-E879.

27. Andrade AF, Paiva WS, Soares MS, et al. Classification and management of mild head trauma. Int J Gen Med. 2011;4: $175-179$.

28. Syed AT, Lone NA, Wani MA, Bhat AS. Clinical management of patients with minor head injuries. Int J Health Sci (Qassim). 2007;1(1):131-140.

29. Martin GT. Acute brain trauma. Ann R Coll Surg Engl. 2016; 98(1):6-10.

30. Baker CC, Oppenheimer L, Stephens B, et al. Epidemiology of trauma deaths. Am J Surg. 1980;140(1):144-150.

\section{Disclosures}

The authors report no conflict of interest concerning the materials or methods used in this study or the findings specified in this paper.

\section{Author Contributions}

Conception and design: Patel, Kelly, Yengo-Kahn. Acquisition of data: Patel, Wolfson, Dawoud, Ahluwalia. Analysis and interpretation of data: Patel, Kelly, Yengo-Kahn, Wolfson. Drafting the article: Patel, Kelly, Yengo-Kahn. Critically revising the article: Patel, Kelly, Yengo-Kahn, Wolfson, Guillamondegui, Bonfield. Reviewed submitted version of manuscript: all authors. Approved the final version of the manuscript on behalf of all authors: Patel. Statistical analysis: Kelly, Yengo-Kahn, Wolfson. Administrative/ technical/material support: Kelly, Yengo-Kahn. Study supervision: Kelly, Yengo-Kahn, Guillamondegui, Bonfield.

\section{Supplemental Information}

\section{Previous Presentations}

Preliminary data from this manuscript were presented virtually at the AANS Annual Scientific Meeting, April 25-29, 2020.

\section{Current Affiliations}

Dr. Wolfson: Department of Neurological Surgery, Rush University Medical Center, Chicago, IL.

\section{Correspondence}

Pious D. Patel: Vanderbilt University School of Medicine, Nashville, TN. pious.d.patel@vanderbilt.edu. 